\title{
Reconfiguration of Dominating Sets
}

\author{
Akira Suzuki $^{1 \star}$, Amer E. Mouawad ${ }^{2 \star \star}$, and Naomi Nishimura ${ }^{2 \star \star}$ \\ 1 Graduate School of Information Sciences, Tohoku University \\ Aoba-yama 6-6-05, Aoba-ku, Sendai, 980-8579, Japan. \\ a.suzuki@ecei.tohoku.ac.jp \\ 2 David R. Cheriton School of Computer Science \\ University of Waterloo, Waterloo, Ontario, Canada. \\ \{aabdomou, nishi\}@uwaterloo.ca
}

\begin{abstract}
We explore a reconfiguration version of the dominating set problem, where a dominating set in a graph $G$ is a set $S$ of vertices such that each vertex is either in $S$ or has a neighbour in $S$. In a reconfiguration problem, the goal is to determine whether there exists a sequence of feasible solutions connecting given feasible solutions $s$ and $t$ such that each pair of consecutive solutions is adjacent according to a specified adjacency relation. Two dominating sets are adjacent if one can be formed from the other by the addition or deletion of a single vertex.

For various values of $k$, we consider properties of $D_{k}(G)$, the graph consisting of a vertex for each dominating set of size at most $k$ and edges specified by the adjacency relation. Addressing an open question posed by Haas and Seyffarth, we demonstrate that $D_{\Gamma(G)+1}(G)$ is not necessarily connected, for $\Gamma(G)$ the maximum cardinality of a minimal dominating set in $G$. The result holds even when graphs are constrained to be planar, of bounded tree-width, or $b$-partite for $b \geq 3$. Moreover, we construct an infinite family of graphs such that $D_{\gamma(G)+1}(G)$ has exponential diameter, for $\gamma(G)$ the minimum size of a dominating set. On the positive side, we show that $D_{n-m}(G)$ is connected and of linear diameter for any graph $G$ on $n$ vertices having at least $m+1$ independent edges.
\end{abstract}

\section{Introduction}

The reconfiguration version of a problem determines whether it is possible to transform one feasible solution $s$ into a target feasible solution $t$ in a step-bystep manner (a reconfiguration) such that each intermediate solution is also feasible. The study of such problems has received considerable attention in recent literature $8[9] 13 \mid 15] 16$ and is interesting for a variety of reasons. From an algorithmic standpoint, reconfiguration models dynamic situations in which we seek to transform a solution into a more desirable one, maintaining feasibility during the process. Reconfiguration also models questions of evolution; it can represent

\footnotetext{
^ Research supported by JSPS Grant-in-Aid for Scientific Research, Grant Number 24.3660

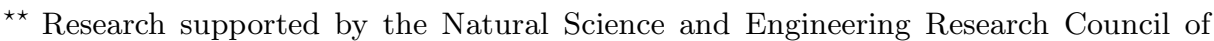
Canada.
} 
the evolution of a genotype where only individual mutations are allowed and all genotypes must satisfy a certain fitness threshold, i.e. be feasible. Moreover, the study of reconfiguration yields insights into the structure of the solution space of the underlying problem, crucial for the design of efficient algorithms. In fact, one of the initial motivations behind such questions was to study the performance of heuristics 9 and random sampling methods 4, where connectivity and other properties of the solution space play a crucial role. Even though reconfiguration gained popularity in the last decade or so, the notion of exploring the solution space of a given problem has been previously considered in numerous settings. One such example is the work of Mayr and Plaxton [18, where the authors consider the problem of transforming one minimum spanning tree of a weighted graph into another by a sequence of edge swaps.

Some of the problems for which the reconfiguration version has been studied include vertex colouring [1|3|4|6|5], list edge-colouring [14, list L(2,1)-labeling [15], block puzzles [11], independent set [11]13], clique, set cover, integer programming, matching, spanning tree, matroid bases [13], satisfiability [9], shortest path [2]16, subset sum [12], dominating set [1019], odd cycle transversal, feedback vertex set, and hitting set 19 . For most NP-complete problems, the reconfiguration version has been shown to be PSPACE-complete [13]14]17, while for some problems in $\mathbf{P}$, the reconfiguration question could be either in $\mathbf{P} 13$ or PSPACE-complete [2].

The problem of transforming input $s$ into input $t$ can be viewed as the problem of determining if there is a path from $s$ to $t$ in a graph representing feasible solutions. Such a path is called a reconfiguration sequence. For the problem of dominating set, the $k$-dominating graph, defined formally in Section 2, consists of a node for each feasible solution and an edge for each pair of solutions that differ by a single vertex. Finding an $s$ - $t$ path in this graph has been shown to be $\mathbf{W}[\mathbf{2}]$-hard [19], and hence not likely to yield even a fixed-parameter tractable algorithm [7].

Although having received less attention than the $s$ - $t$ path problem, other characteristics of the solution graph have been studied. Determining the diameter of the reconfiguration graph will result in an upper bound on the length of any reconfiguration sequence. For a problem such as colouring, one can determine the mixing number, the minimum number of colours needed to ensure that the entire graph is connected; such a number has been obtained for the problem of list edge-colouring on trees [14].

In previous work on reconfiguration of dominating sets, Haas and Seyffarth [10] considered the connectivity of the graph of solutions of size at most $k$, for various values of $k$ relative to $n$, the number of vertices in the input graph $G$. They demonstrated that the graph is connected when $k=n-1$ and $G$ has at least two independent edges, or when $k$ is one greater than the maximum cardinality of a minimal dominating set and $G$ is non-trivially bipartite or chordal. They left as an open question, answered negatively here, whether the latter results could be extended to all graphs. 
In this paper we extend previous work by showing in Section 3 that the solution graph is connected and of linear diameter for $k=n-m$ for any input graph with at least $m+1$ independent edges, for any nonnegative integer $m$. In Section 4, we give a series of counterexamples demonstrating that $D_{\Gamma(G)+1}(G)$ is not guaranteed to be connected for planar graphs, graphs of bounded treewidth, or $b$-partite graphs for $b \geq 3$. In Section 5 , we pose and answer a question about the diameter of $D_{\gamma(G)+1}(G)$ by showing that there is an infinite family of graphs of exponential diameter.

\section{Preliminaries}

We assume that each $G$ is a simple, undirected graph on $n$ vertices with vertex set $V(G)$ and edge set $E(G)$. The diameter of $G$ is the maximum over all pairs of vertices $u$ and $v$ in $V(G)$ of the length of the shortest path between $u$ and $v$.

A set $S \subseteq V(G)$ is a dominating set of $G$ if and only if every vertex in $V(G) \backslash S$ is adjacent to a vertex in $S$. The minimum cardinality of any dominating set of $G$ is denoted by $\gamma(G)$. Similarly, $\Gamma(G)$ is the maximum cardinality of any minimal dominating set in $G$.

For a vertex $u \in V(G)$ and a dominating set $S$ of $G$, we say $u$ is dominated by $v \in S$ if $u \notin S$ and $u$ is adjacent to $v$. For a vertex $v$ in a dominating set $S$, a private neighbour of $v$ is a vertex dominated by $v$ and not dominated by any other vertex in $S$; the private neighbourhood of $v$ is the set of private neighbours of $v$. A vertex $v$ in a dominating set $S$ is deletable if $S \backslash\{v\}$ is also a dominating set of $G$.

Fact $1 A$ vertex $v$ is deletable if and only if $v$ has at least one neighbour in $S$ and $v$ has no private neighbour.

Given a graph $G$ and a positive integer $k$, we consider the $k$-dominating graph of $G, D_{k}(G)$, such that each vertex in $V\left(D_{k}(G)\right)$ corresponds to a dominating set of $G$ of cardinality at most $k$. Two vertices are adjacent in $D_{k}(G)$ if and only if the corresponding dominating sets differ by either the addition or the deletion of a single vertex; each such operation is a reconfiguration step. Formally, if $A$ and $B$ are dominating sets of $G$ of cardinality at most $k$, then there exists an edge between $A$ and $B$ if and only if there exists a vertex $u \in V(G)$ such that $(A \backslash B) \cup(B \backslash A)=\{u\}$. We refer to vertices in $G$ using lower case letters (e.g. $u, v)$ and to the vertices in $D_{k}(G)$, and by extension their associated dominating sets, using upper case letters (e.g. $A, B$ ). We write $A \leftrightarrow B$ if there exists a path in $D_{k}(G)$ joining $A$ and $B$. The following fact is a consequence of our ability to add vertices as needed to form $B$ from $A$.

Fact 2 If $A \subseteq B$, then $A \leftrightarrow B$ and $B \leftrightarrow A$.

\section{Graphs with $\boldsymbol{m}+\mathbf{1}$ independent edges}

Theorem 1. For any nonnegative integer $m$, if $G$ has at least $m+1$ independent edges, then $D_{n-m}(G)$ is connected for $n=|V(G)|$. 
Proof. For $G$ a graph with $m+1$ independent edges $I=\left\{\left\{u_{i}, w_{i}\right\} \mid 0 \leq i \leq m\right\}$, we define $U=\left\{u_{i} \mid 0 \leq i \leq m\right\}, W=\left\{w_{i} \mid 0 \leq i \leq m\right\}$, and the set of outsiders $R=V(G) \backslash(U \cup W)$.

Using any dominating set $S$ of $G$, we can partition $I$ as follows: edge $\left\{u_{i}, w_{i}\right\}$, $0 \leq i \leq m$, is clean if neither $u_{i}$ nor $w_{i}$ is in $S, u$-odd if $u_{i} \in S$ but $w_{i} \notin S, w$-odd if $w_{i} \in S$ but $u_{i} \notin S$, odd if $\left\{u_{i}, w_{i}\right\}$ is $u$-odd or $w$-odd, and even if $\left\{u_{i}, w_{i}\right\} \subseteq S$. We use clean $(S)$ and $\operatorname{odd}(S)$, respectively, to denote the numbers of clean and odd edges for $S$. Similarly, we let $u$-odd $(S)$ and $w$-odd $(S)$ denote the numbers of $u$-odd and $w$-odd edges for $S$. In the example graph shown in Figure1, $m+1=7$ and $R=\emptyset$. There is a single clean edge, namely $\left\{u_{1}, w_{1}\right\}$, three $w$-odd edges, two $u$-odd edges, and a single even edge.

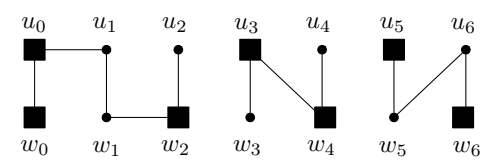

Fig. 1. Vertices in $S$ are marked with squares

It suffices to show that for $S$ an arbitrary dominating set of $G$ such that $|S| \leq n-m, S \leftrightarrow N$ for $N=V(G) \backslash W ; N$ is clearly a dominating set as each vertex $w_{i} \in W=V(G) \backslash N$ is dominated by $u_{i}$. By Fact 2, for $S^{\prime}$ a dominating set of $G$ such that $S^{\prime} \supseteq S$ and $\left|S^{\prime}\right|=n-m$, since $S^{\prime}$ is a superset of $S$, then $S \leftrightarrow S^{\prime}$. The reconfiguration from $S^{\prime}$ to $N$ can be broken into three stages. In the first stage, for a dominating set $S_{0}$ with no clean edges, we show $S^{\prime} \leftrightarrow S_{0}$ by repeatedly decrementing the number of clean edges $\left(u_{i}\right.$ or $w_{i}$ is added to the dominating set for some $0 \leq i \leq m)$. In the second stage, for $T_{m}$ with $m u$-odd edges and one even edge, we show $S_{0} \leftrightarrow T_{m}$ by repeatedly incrementing the number of $u$-odd edges. Finally, we observe that deleting the single remaining element in $T_{m} \cap W$ yields $T_{m} \leftrightarrow N$.

In stage 1, for $x=\operatorname{clean}\left(S^{\prime}\right)$, we show that $S^{\prime}=S_{x} \leftrightarrow S_{x-1} \leftrightarrow S_{x-2} \leftrightarrow \ldots \leftrightarrow$ $S_{0}$ where for each $0 \leq j \leq x, S_{j}$ is a dominating set of $G$ such that $\left|S_{j}\right|=n-m$ and clean $\left(S_{j}\right)=j$. To show that $S_{a} \leftrightarrow S_{a-1}$ for arbitrary $1 \leq a \leq x$, we prove that there is a deletable vertex in some even edge and hence a vertex in a clean edge can be added in the next reconfiguration step. For $b=\operatorname{odd}\left(S_{a}\right)$, the set $E$ of vertices in even edges is of size $2((m+1)-a-b)$. Since each vertex in $E$ has a neighbour in $S_{a}$, if at least one vertex in $E$ does not have a private neighbour, then $E$ contains a deletable vertex (Fact 1).

The $m$ vertices in $V(G) \backslash S_{a}$ are the only possible candidates to be private neighbours. Of these, the $b$ vertices of $V(G) \backslash S_{a}$ in odd edges cannot be private neighbours of vertices in $E$, as each is the neighbour of a vertex in $S_{a} \backslash E$ (the other endpoint of the edge). The number of remaining candidates, $m-b$, is smaller than the number of vertices in $E ; m \geq 2 a+b$ as the vertices of $V(G) \backslash S_{a}$ must contain both endpoints of any clean edge and one endpoint for any odd 
edge. Hence, there exists at least one deletable vertex in $E$. When we delete such a vertex and add an arbitrary endpoint of a clean edge, the clean edge becomes an odd edge and the number of clean edges decreases. We can therefore reconfigure from $S_{a}$ to the desired dominating set, and by applying the same argument $a$ times, to $S_{0}$.

In the second stage we show that for $y=u$-odd $\left(S_{0}\right), S_{0}=T_{y} \leftrightarrow T_{y+1} \leftrightarrow$ $T_{y+2} \leftrightarrow \ldots \leftrightarrow T_{m}$ where for each $y \leq j \leq m, T_{j}$ is a dominating set of $G$ such that $\left|T_{j}\right|=n-m$, clean $\left(T_{j}\right)=0$, and $u$-odd $\left(T_{j}\right)=j$. To show that $T_{c} \leftrightarrow T_{c+1}$ for arbitrary $y \leq c \leq m-1$, we use a counting argument to find a vertex in an even edge that is in $W$ and deletable; in one reconfiguration step the vertex is deleted, increasing the number of $u$-odd edges, and in the next reconfiguration step an arbitrary vertex in $R$ or in a $w$-odd edge is added to the dominating set. We let $d=w$-odd $\left(T_{c}\right)$ (i.e. the number of $w$-odd edges for $T_{c}$ ) and observe that since there are $c u$-odd edges, $d w$-odd edges, and no clean edges, there exist $(m+1)-c-d$ even edges. We define $E_{w}$ to be the set of vertices in $W$ that are in the even edges, and observe that each has a neighbour in $T_{c}$; a vertex in $E_{w}$ will be deletable if it does not have a private neighbour.

Of the $m$ vertices in $V(G) \backslash T_{c}$, only those in $R$ are candidates to be private neighbours of vertices in $E_{w}$, as each vertex in an odd edge has a neighbour in $T_{c}$. As there are $c u$-odd edges and $d w$-odd edges, the total number of vertices in $R \cap V(G) \backslash T_{c}$ is $m-c-d$. Since this is smaller than the number of vertices in $E_{w}$, at least one vertex in $E_{w}$ must be deletable. When we delete such a vertex from $T_{c}$ and in the next step add an arbitrary vertex from the outsiders or $w$ odd edges, the even edge becomes a $u$-odd edge and the number of $u$-odd edges increases. Note that we can always find such a vertex since there are $m-c-d$ outsiders, $d w$-odd edges, and $c \leq m-1$. Hence, we can reconfigure from $T_{c}$ to $T_{c+1}$, and by $m-c$ repetitions, to $T_{m}$.

Corollary 1 results from the length of the reconfiguration sequence formed in Theorem 11 reconfiguring to $S^{\prime}$ can be achieved in at most $n-m$ steps, and stages 1 and 2 require at most $2 m$ steps each, as $m \in O(n)$ is at most the numbers of clean and $u$-odd edges. Theorem 2 shows that Theorem 1 is tight.

Corollary 1. The diameter of $D_{n-m}(G)$ is in $O(n)$ for $G$ a graph with $m+1$ independent edges.

Theorem 2. For any nonnegative integer $m$, there exists a graph $G_{m}$ with $m$ independent edges such that $D_{n-m}\left(G_{m}\right)$ is not connected.

Proof. Let $G_{m}$ be a path on $n=2 m$ vertices. Clearly, $G_{m}$ has $m$ disjoint edges, $n-m=2 m-m=m$, and $D_{n-m}\left(G_{m}\right)=D_{m}\left(G_{m}\right)$. We let $S$ be a dominating set of $G_{m}$ such that $|S| \geq m+1$. At least one vertex in $S$ must have all its neighbors in $S$ and is therefore deletable. It follows that $\Gamma\left(G_{m}\right)=m$ and $D_{n-m}\left(G_{m}\right)=$ $D_{m}\left(G_{m}\right)=D_{\Gamma\left(G_{m}\right)}\left(G_{m}\right)$ which is not connected by the result of Haas and Seyffarth [10, Lemma 3]. 


\section{$4 D_{\Gamma(G)+1}(G)$ may not be connected}

In this section we demonstrate that $D_{\Gamma(G)+1}(G)$ is not connected for an infinite family of graphs $G_{(d, b)}$ for all positive integers $b \geq 3$ and $d \geq 2$, where graph $G_{(d, b)}$ is constructed from $d+1$ cliques of size $b$. We demonstrate using the graph $G_{(4,3)}$ as shown in part (a) of Figure 2, consisting of fifteen vertices partitioned into five cliques of size 3: the outer clique $C_{0}$, consisting of the top, left, and right outer vertices $o_{1}, o_{2}$, and $o_{3}$, and the four inner cliques $C_{1}$ through $C_{4}$, ordered from left to right. We use $v_{(i, 1)}, v_{(i, 2)}$, and $v_{(i, 3)}$ to denote the top, left, and right vertices in clique $C_{i}, 1 \leq i \leq 4$. More generally, a graph $G_{(d, b)}$ has $d+1 b$-cliques $C_{i}$ for $0 \leq i \leq d$. The clique $C_{0}$ consists of outer vertices $o_{j}$ for $1 \leq j \leq b$, and for each inner clique $C_{i}, 1 \leq i \leq d$ and each $1 \leq j \leq b$, there exists an edge $\left\{o_{j}, v_{(i, j)}\right\}$.

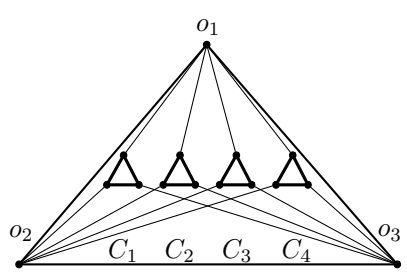

(a)

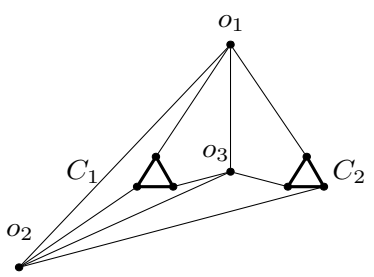

(b)

Fig. 2. Counterexamples for (a) general and (b) planar graphs

For any $1 \leq j \leq b$ a dominating set does not contain $o_{j}$, then the vertices $v_{(i, j)}$ of the inner cliques must be dominated by vertices in the inner cliques (hence Fact (3). In addition, the outer vertex $o_{j}$ can be dominated only by another outer vertex or some vertex $v_{(i, j)}, 1 \leq i \leq d$ (hence Fact 4 ).

Fact 3 Any dominating set that does not contain all of the outer vertices must contain at least one vertex from each of the inner cliques.

Fact 4 Any dominating set that does not contain any outer vertex must contain at least one vertex of the form $v_{(\cdot, j)}$ for each $1 \leq j \leq b$.

Lemma 1. For each graph $G_{(d, b)}$ as defined above, $\Gamma\left(G_{(d, b)}\right)=d+b-2$.

Proof. We first demonstrate that there is a minimal dominating set of size $d+$ $b-2$, consisting of $\left\{v_{(1, j)} \mid 2 \leq j \leq b\right\} \cup\left\{v_{(i, 1)} \mid 2 \leq i \leq d\right\}$; the first set dominates $b-1$ of the outer vertices and the first inner clique and the second set dominates $o_{1}$ and the rest of the inner cliques. The dominating set is minimal, as the removal of any vertex $v_{(1, j)}, 2 \leq j \leq b$, would leave vertex $o_{j}$ with no neighbour in the dominating set and the removal of any $v_{(i, 1)}, 2 \leq i \leq d$, would leave $\left\{v_{(i, j)} \mid 1 \leq j \leq b\right\}$ with no neighbour in the dominating set. 
By Fact 3, any dominating set that does not contain all outer vertices must contain at least one vertex in each of the $d$ inner cliques. Since the outer vertices form a minimal dominating set, any other minimal dominating set must contain at least one vertex from each of the inner cliques.

We now consider any dominating set $S$ of size at least $d+b-1$ containing one vertex for each inner clique and show that it is not minimal. If $S$ contains at least one outer vertex, we can find a smaller dominating set by removing all but the outer vertex and one vertex for each inner clique, yielding a total of $d+1<d+b-1$ vertices (since $b \geq 3$ ). Now suppose that $S$ consists entirely of inner vertices; by Fact $4 S$ contains at least one vertex of the form $v_{(\cdot, j)}$ for each $1 \leq j \leq b$. Moreover, for at least one value $1 \leq j^{\prime} \leq b$, there exists more than one vertex of the form $v_{\left(\cdot, j^{\prime}\right)}$ as $d+b-1>b$. This allows us to choose $b$ vertices of the form $v_{(\cdot, j)}$ for each $1 \leq j \leq b$ that dominate at least two inner cliques as well as all outer vertices. By selecting one member of $S$ from each of the remaining $d-2$ inner cliques, we form a dominating set of size $d+b-2<d+b-1$, proving that $S$ is not minimal.

Theorem 3. There exists an infinite family of graphs such that for each $G$ in the family, $D_{\Gamma(G)+1}(G)$ is not connected.

Proof. For any positive integers $b \geq 3$ and $d \geq 2$, we show that there is no path between dominating sets $A$ to $B$ in $D_{d+b-1}\left(G_{(d, b)}\right)$, where $A$ consists of the vertices in the outer clique and $B$ consists of $\left\{v_{(i, \ell)} \mid 1 \leq i \leq d, 1 \leq \ell \leq b, i \equiv\right.$ $\ell(\bmod b)\}$;

By Fact 3 before we can delete any of the vertices in $A$, we need to add one vertex from each of the inner cliques, resulting in a dominating set of size $d+b=\Gamma\left(G_{(d, b)}\right)+2$. As there is no such vertex in our graph, there is no way to connect $A$ and $B$.

Each graph $G_{(d, b)}$ constructed for Theorem 3 is a $b$-partite graph; we can partition the vertices into $b$ independent sets, where the $j$ th set, $1 \leq j \leq b$ is defined as $\left\{v_{(i, j)} \mid 1 \leq i \leq d\right\} \cup\left\{o_{i} \mid 1 \leq i \leq d, i \equiv j+1(\bmod b)\right\}$. Moreover, we can form a tree decomposition of width $2 b-1$ of $G_{(d, b)}$, for all positive integers $b \geq 3$ and $d \geq b$, by creating bags with the vertices of the inner cliques and adding all outer vertices to each bag.

Corollary 2. For every positive integer $b \geq 3$, there exists an infinite family of graphs of tree-width $2 b-1$ such that for each $G$ in the family, $D_{\Gamma(G)+1}(G)$ is not connected, and an infinite family of b-partite graphs such that for each $G$ in the family, $D_{\Gamma(G)+1}(G)$ is not connected.

Theorem 3 does not preclude the possibility that when restricted to planar graphs or any other graph class that excludes $G_{(d, b)}, D_{\Gamma(G)+1}(G)$ is connected. However, the next corollary follows directly from the fact that $G_{(2,3)}$ is planar (part (b) of Figure 2).

Corollary 3. There exists a planar graph $G$ for which $D_{\Gamma(G)+1}(G)$ is not connected. 


\section{On the diameter of $D_{k}(G)$}

In this section, we obtain a lower bound on the diameter of the $k$-dominating graph of a family of graphs $G_{n}$. We describe $G_{n}$ in terms of several component subgraphs, each playing a role in forcing the reconfiguration of dominating sets.

A linkage gadget (part (a), Figure 3) consists of five vertices, the external vertices (or endpoints) $e_{1}$ and $e_{2}$, and the internal vertices $i_{1}, i_{2}$, and $i_{3}$. The external vertices are adjacent to each internal vertex as well as to each other; the following results from the internal vertices having degree two:

Fact 5 In a linkage gadget, the minimum dominating sets of size one are $\left\{e_{1}\right\}$ and $\left\{e_{2}\right\}$. Any dominating set containing an internal vertex must contain at least two vertices. Any dominating set in a graph containing $m$ vertex-disjoint linkage gadgets with all internal vertices having degree exactly two must contain at least one vertex in each linkage gadget.

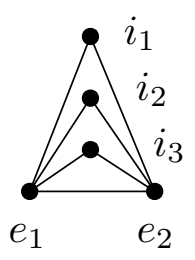

(a)

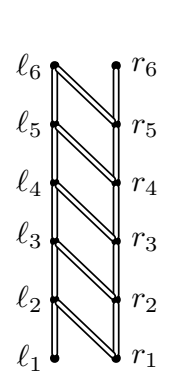

(b)

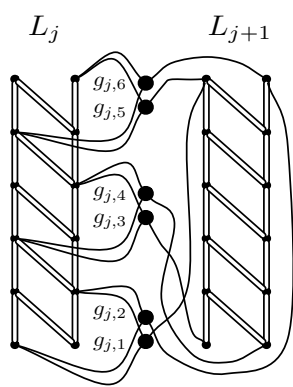

(c)

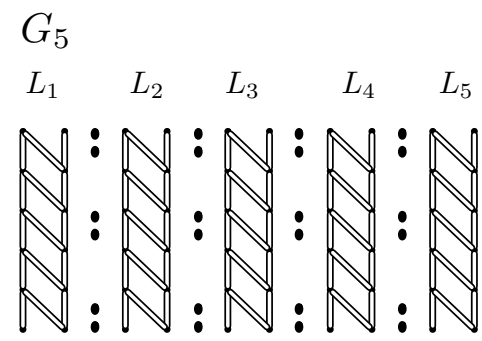

(d)

Fig. 3. Parts of the construction

A ladder (part (b) of Figure 3, linkages shown as double edges) is a graph consisting of twelve ladder vertices paired into six rungs, where rung $i$ consists of the vertices $\ell_{i}$ and $r_{i}$ for $1 \leq i \leq 6$, as well as the 45 internal vertices of fifteen linkage gadgets. Each linkage gadget is associated with a pair of ladder vertices, where the ladder vertices are the external vertices in the linkage gadget. The fifteen pairs are as follows: ten vertical pairs $\left\{\ell_{i}, \ell_{i+1}\right\}$ and $\left\{r_{i}, r_{i+1}\right\}$ for $1 \leq i \leq 5$, and five cross pairs $\left\{\ell_{i+1}, r_{i}\right\}$ for $1 \leq i \leq 5$. For convenience, we refer to vertices $\ell_{i}, 1 \leq i \leq 6$ and the associated linkage gadgets as the left side of the ladder and to vertices $r_{i}, 1 \leq i \leq 6$ and the associated linkage gadgets as the right side of the ladder, or collectively as the sides of the ladder.

The graph $G_{n}$ consists of $n$ ladders $L_{1}$ through $L_{n}$ and $n-1$ sets of gluing vertices, where each set consists of three clusters of two vertices each. For $\ell_{j, i}$ and $r_{j, i}, 1 \leq i \leq 6$, the ladder vertices of ladder $L_{j}$, and $g_{j, 1}$ through $g_{j, 6}$ the 
gluing vertices that join ladders $L_{j}$ and $L_{j+1}$, we have the following connections for $1 \leq j \leq n-1$ :

- Edges connecting the bottom cluster to the bottom two rungs of ladder $L_{j}$ and the top rung of ladder $L_{j+1}:\left\{\ell_{j, 1}, g_{j, 1}\right\},\left\{\ell_{j, 1}, g_{j, 2}\right\},\left\{r_{j, 2}, g_{j, 1}\right\},\left\{r_{j, 2}, g_{j, 2}\right\}$, $\left\{\ell_{j+1,6}, g_{j, 1}\right\},\left\{r_{j+1,6}, g_{j, 2}\right\}$

- Edges connecting the middle cluster to the middle two rungs of ladder $L_{j}$ and the bottom rung of ladder $L_{j+1}:\left\{\ell_{j, 3}, g_{j, 3}\right\},\left\{\ell_{j, 3}, g_{j, 4}\right\},\left\{r_{j, 4}, g_{j, 3}\right\}$, $\left\{r_{j, 4}, g_{j, 4}\right\},\left\{\ell_{j+1,1}, g_{j, 3}\right\},\left\{r_{j+1,1}, g_{j, 4}\right\}$

- Edges connecting the top cluster to the top two rungs of ladder $L_{j}$ and the top rung of ladder $L_{j+1}:\left\{\ell_{j, 5}, g_{j, 5}\right\},\left\{\ell_{j, 5}, g_{j, 6}\right\},\left\{r_{j, 6}, g_{j, 5}\right\},\left\{r_{j, 6}, g_{j, 6}\right\}$, $\left\{\ell_{j+1,6}, g_{j, 5}\right\},\left\{r_{j+1,6}, g_{j, 6}\right\}$

Figure 3 parts (c) and (d) show details of the construction of $G_{n}$; they depict, respectively, two consecutive ladders and $G_{5}$, both with linkages represented as double edges. When clear from context, we sometimes use single subscripts instead of double subscripts to refer to the vertices of a single ladder.

We let $\mathcal{D}=\left\{\left\{\ell_{(j, 2 i-1)}, \ell_{(j, 2 i)}\right\},\left\{r_{(j, 2 i-1)}, r_{(j, 2 i)}\right\} \mid 1 \leq i \leq 3,1 \leq j \leq n\right\}$ denote a set of $6 n$ pairs in $G_{n}$; the corresponding linkage gadgets are vertexdisjoint. Then Fact 5 implies the following:

Fact 6 Any dominating set $S$ of $G_{n}$ must contain at least one vertex of each of the linkage gadgets for vertical pairs in the set $\mathcal{D}$ and hence is of size at least $6 n$; if $S$ contains an internal vertex, then $|S|>6 n$.

Choosing an arbitrary external vertex for each vertical pair does not guarantee that all vertices on the side of a ladder are dominated; for example, the set $\left\{\ell_{i} \mid i \in\{1,4,5\}\right\}$ does not dominate the internal vertices in the vertical pair $\left\{\ell_{2}, \ell_{3}\right\}$. Choices that do not leave such gaps form the set $\mathcal{C}=\left\{\mathcal{C}_{i} \mid 1 \leq i \leq 4\right\}$ where $\mathcal{C}_{1}=\{1,3,5\}, \mathcal{C}_{2}=\{2,3,5\}, \mathcal{C}_{3}=\{2,4,5\}$, and $\mathcal{C}_{4}=\{2,4,6\}$.

Fact 7 In any dominating set $S$ of size $6 n$ and in any ladder $L$ in $G_{n}$, the restriction of $S$ to $L$ must be of the form $S_{i}$ for some $1 \leq i \leq 7$, as illustrated in Figure 4.

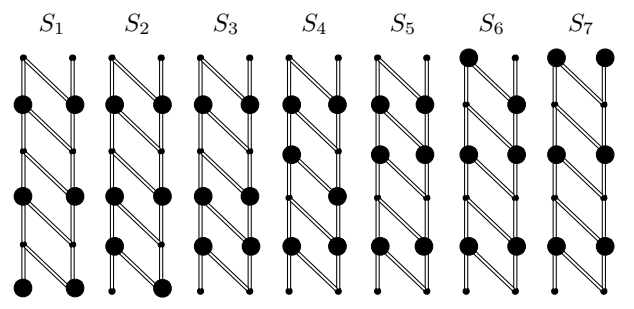

Fig. 4. Minimum dominating sets for $G_{1}$ 
Proof. Fact 6 implies that the only choices for the left (right) vertices are $\left\{\ell_{i} \mid\right.$ $\left.i \in \mathcal{C}_{j}\right\}\left(\left\{r_{i} \mid i \in \mathcal{C}_{j}\right\}\right)$ for $1 \leq j \leq 4$. The sets $S_{i}, 1 \leq i \leq 7$, are the only combinations of these choices that dominate all the internal vertices in the cross pairs.

We say that ladder $L_{j}$ is in state $S_{i}$ if the restriction of the dominating set to $L_{j}$ is of the form $S_{i}$, for $1 \leq j \leq n$ and $1 \leq i \leq 7$.

The exponential lower bound in Theorem 4 is based on counting how many times each ladder is modified from $S_{1}$ to $S_{7}$ or vice versa; we say ladder $L_{j}$ undergoes a switch for each such modification. We first focus on a single ladder.

Fact 8 For $S$ a dominating set of $G_{1}$, a vertex $v \in S$ is deletable if and only if either $v$ is the internal vertex of a linkage gadget one of whose external vertices is in $S$, or for every linkage gadget containing $v$ as an external vertex, either the other external vertex is also in $S$ or all internal vertices are in $S$.

Lemma 2. In $D_{\gamma\left(G_{1}\right)+1}\left(G_{1}\right)$ there is a single reconfiguration sequence between $S_{1}$ and $S_{7}$, of length 12 .

Proof. We define $P$ to be the path in the graph corresponding to the reconfiguration sequence $S_{1} \leftrightarrow S_{1} \cup\left\{\ell_{2}\right\} \leftrightarrow S_{2} \leftrightarrow S_{2} \cup\left\{r_{2}\right\} \leftrightarrow S_{3} \leftrightarrow S_{3} \cup\left\{\ell_{4}\right\} \leftrightarrow S_{4} \leftrightarrow$ $S_{4} \cup\left\{r_{4}\right\} \leftrightarrow S_{5} \leftrightarrow S_{5} \cup\left\{\ell_{6}\right\} \leftrightarrow S_{6} \leftrightarrow S_{6} \cup\left\{r_{6}\right\} \leftrightarrow S_{7}$ and demonstrate that there is no shorter path between $S_{1}$ and $S_{7}$.

By Facts 7 and 6. $G_{1}$ has exactly seven dominating sets of size six, and any dominating set $S$ of size seven contains two vertices from one vertical pair $d$ in $\mathcal{D}$ and one from each of the remaining five. The neighbours of $S$ in $D_{\gamma\left(G_{1}\right)+1}\left(G_{1}\right)$ are the vertices corresponding to the sets $S_{i}, 1 \leq i \leq 7$, obtained by deleting a single vertex of $S$. The number of neighbours is thus at most two, depending on which, if any, vertices in $d$ are deletable.

If at least one of the vertices of $S$ in $d$ is an internal vertex, then at most one vertex satisfies the first condition in Fact 8 . Thus, for $S$ to have two neighbours, there must be a ladder vertex that satisfies the second condition of Fact 8 , which by inspection of Figure 4 can be seen to be false.

If instead $d$ contains two ladder vertices, in order for $S$ to have two neighbours, the four ladder vertices on the side containing $d$ must correspond to the union of two of the sets in $\mathcal{C}$. There are only three such unions, $\mathcal{C}_{1} \cup \mathcal{C}_{2}, \mathcal{C}_{2} \cup \mathcal{C}_{3}$, and $\mathcal{C}_{3} \cup \mathcal{C}_{4}$, which implies that the only pairs with common neighbours are $\left\{S_{i}, S_{i+1}\right\}$ for $1 \leq i \leq 6$, as needed to complete the proof.

For $n>2$, we cannot reconfigure ladders independently from each other, as we need to ensure that all gluing vertices are dominated. For consecutive ladders $L_{j}$ and $L_{j+1}$, any cluster that is not dominated by $L_{j}$ must be dominated by $L_{j+1}$; the bottom, middle, and top clusters are not dominated by any vertex in $S_{2}, S_{4}$, and $S_{6}$, respectively.

Fact 9 In any dominating set $S$ of $G_{n}$, for any $1 \leq j<n$, if $L_{j}$ is in state $S_{2}$, then $L_{j+1}$ is in state $S_{7}$; if $L_{j}$ is in state $S_{4}$, then $L_{j+1}$ is in state $S_{1}$; and if $L_{j}$ is in state $S_{6}$, then $L_{j+1}$ is in state $S_{7}$. 
Lemma 3. For any reconfiguration sequence in which $L_{j}$ and $L_{j+1}$ are initially both in state $S_{1}$, if $L_{j}$ undergoes $p$ switches then $L_{j+1}$ must undergo at least $2 p+1$ switches.

Proof. We use a simple counting argument. When $p=1$, the result follows immediately from Fact 9 since $L_{j}$ can only reach state $S_{7}$ if $L_{j+1}$ is reconfigured from $S_{1}$ to $S_{7}$ to $S_{1}$ and finally back to $S_{7}$. After the first switch of $L_{j}$, both ladders are in state $S_{7}$.

For any subsequent switch of $L_{j}, L_{j}$ starts in state $S_{7}$ because for $L_{j}$ to reach $S_{1}$ from $S_{2}$ or to reach $S_{7}$ from $S_{6}$, by Fact $9 L_{j+1}$ must have been in $S_{7}$. Since by definition $L_{j}$ starts in $S_{1}$ or $S_{7}$, to enable $L_{j}$ to undergo a switch, $L_{j+1}$ will have to undergo at least two switches, namely $S_{7}$ to $S_{1}$ and back to $S_{7}$.

Theorem 4. For $S$ a dominating set of $G_{n}$ such that every ladder of $G_{n}$ is in state $S_{1}$ and $T$ a dominating set of $G_{n}$ such that every ladder of $G_{n}$ is in state $S_{7}$, the length of any reconfiguration sequence between $S$ and $T$ is at least $12\left(2^{n+1}-n-2\right)$.

Proof. We first observe that Lemma 2 implies that the switch of any ladder requires at least twelve reconfiguration steps; since the vertex associated with a dominating set containing a gluing vertex will have degree at most one in the $k$-dominating graph, there are no shortcuts formed.

To reconfigure from $S$ to $T$, ladder $L_{1}$ must undergo at least one switch. By Lemma 3. ladder $L_{2}$ will undergo at least $3=2^{2}-1$ switches, hence $2^{j}-1$ switches for ladder $L_{j}, 1 \leq j \leq n$. Since each switch requires twelve steps, the total number of steps is thus at least $12 \sum_{i=1}^{n}\left(2^{i}-1\right)=12\left(2^{n+1}-n-2\right)$.

Corollary 4. There exists an infinite family of graphs such that for each graph $G_{n}$ in the family, $D_{\gamma\left(G_{n}\right)+1}\left(G_{n}\right)$ has diameter $\Omega\left(2^{n}\right)$.

\section{Conclusions and future work}

In answering Haas and Seyffarth's question concerning the connectivity of $D_{k}(G)$ for general graphs and $k=\Gamma(G)+1$, we have demonstrated infinite families of planar, bounded treewidth, and $b$-partite graphs for which the $k$-dominating graph is not connected. It remains to be seen whether $k$-dominating graphs are connected for graphs more general than non-trivially bipartite graphs or chordal graphs, and whether $D_{\Gamma(G)+2}(G)$ is connected for all graphs. It would also be useful to know if there is a value of $k$ for which $D_{k}(G)$ is guaranteed not to have exponential diameter. Interestingly, for our connectivity and diameter examples, incrementing the size of the sets by one is sufficient to break the proofs.

\section{References}

1. Marthe Bonamy and Nicolas Bousquet. Recoloring bounded treewidth graphs. In Proc. of the 7th Latin-American Algorithms, Graphs, and Optimization Symp., 2013. 
2. P. Bonsma. The complexity of rerouting shortest paths. In Proc. of Mathematical Foundations of Computer Science, pages 222-233, 2012.

3. P. Bonsma and L. Cereceda. Finding paths between graph colourings: PSPACE-completeness and superpolynomial distances. Theor. Comput. Sci., 410(50):5215-5226, 2009.

4. L. Cereceda, J. van den Heuvel, and M. Johnson. Connectedness of the graph of vertex-colourings. Discrete Math., 308(56):913-919, 2008.

5. L. Cereceda, J. van den Heuvel, and M. Johnson. Finding paths between 3-colorings. J. of Graph Theory, 67(1):69-82, 2011.

6. Luis Cereceda, Jan van den Heuvel, and Matthew Johnson. Mixing 3colourings in bipartite graphs. European J. of Combinatorics, 30(7):15931606, 2009.

7. R. G. Downey and M. R. Fellows. Parameterized complexity. Spring-Verlag, New York, 1997.

8. Gerd Fricke, Sandra Mitchell Hedetniemi, Stephen T. Hedetniemi, and Kevin R. Hutson. $\gamma$-Graphs of Graphs. Discussiones Mathematicae Graph Theory, 31(3):517-531, 2011.

9. P. Gopalan, P. G. Kolaitis, E. N. Maneva, and C.H. Papadimitriou. The connectivity of boolean satisfiability: computational and structural dichotomies. SIAM J. on Computing, 38(6):2330-2355, 2009.

10. R. Haas and K. Seyffarth. The $k$-Dominating Graph. Graphs and Combinatorics, March 2013. Online publication.

11. R. A. Hearn and E. D. Demaine. PSPACE-completeness of sliding-block puzzles and other problems through the nondeterministic constraint logic model of computation. Theor. Comput. Sci., 343(1-2):72-96, 2005.

12. T. Ito and E. D. Demaine. Approximability of the subset sum reconfiguration problem. In Proc. of the 8th Annual Conf. on Theory and Applications of Models of Computation, pages 58-69, 2011.

13. T. Ito, E. D. Demaine, N. J. A. Harvey, C. H. Papadimitriou, Martha Sideri, Ryuhei Uehara, and Yushi Uno. On the complexity of reconfiguration problems. Theor. Comput. Sci., 412(12-14):1054-1065, 2011.

14. T. Ito, M. Kamiński, and E. D. Demaine. Reconfiguration of list edgecolorings in a graph. Discrete Applied Math., 160(15):2199-2207, 2012.

15. T. Ito, K. Kawamura, H. Ono, and X. Zhou. Reconfiguration of list L(2,1)labelings in a graph. In Proc. of the 23rd Int. Symp. on Algorithms and Computation, pages 34-43, 2012.

16. M. Kamiński, P. Medvedev, and M. Milanič. Shortest paths between shortest paths. Theor. Comput. Sci., 412(39):5205-5210, 2011.

17. M. Kamiński, P. Medvedev, and M. Milanič. Complexity of independent set reconfigurability problems. Theor. Comput. Sci., 439:9-15, June 2012.

18. E. W. Mayr and C. G. Plaxton. On the spanning trees of weighted graphs. Combinatorica, 12(4):433-447, 1992.

19. A. E. Mouawad, N. Nishimura, V. Raman, N. Simjour, and A. Suzuki. On the parameterized complexity of reconfiguration problems. In Proc. of the 8th Int. Symp. on Parameterized and Exact Computation, pages 281-294, 2013. 